\section{Anti-TNF therapy can reverse synovial lymphoid neogenesis in patients with RA}

Synovial lymphoid neogenesis (SLN) is a process whereby lymphocyte aggregates, which resemble secondary lymphoid organs, infiltrate the synovial tissue in patients with rheumatoid arthritis (RA) and other autoimmune disorders. These aggregates are often associated with increased tumor necrosis factor (TNF) production and disease severity in RA. Cañete and colleagues studied the clinical and pathological features associated with SLN in patients with RA.

Synovial biopsy samples, taken from the knees of 86 patients with RA (mean age 60.3 years, $67.4 \%$ women), were analyzed. Compared with $\mathrm{SLN}^{-}$patients $(n=44), \mathrm{SLN}^{+}$patients $(n=42)$ had significantly longer disease duration (mean 94.1 months vs 54.1 months, $P=0.0006$ ) and their biopsy specimens contained significantly higher numbers of $\mathrm{CD}^{+} \mathrm{T}$ cells $(P=0.0011)$ and $\mathrm{CD} 20^{+} \mathrm{B}$ cells $(P=0.0004)$. During followup (mean 43 months) patients were treated according to a sequential therapy regimen. Presence of SLN was found to be an independent negative predictor of EULAR response.

A second group of 24 patients, who had failed to respond to previous methotrexate or anti-TNF therapy, received 12 weeks' antiTNF therapy (using a different agent if previous anti-TNF therapy was ineffective). Of the 16 $\mathrm{SLN}^{+}$patients at initial synovial biopsy, 9 (56\%) became $\mathrm{SLN}^{-}$on second biopsy at 12 weeks; this reversal in SLN status was also associated with good clinical responses.

The authors conclude that SLN in patients with RA is a predictor of poor response to therapy; however, SLN positivity is reversed by a short period of anti-TNF therapy in some patients.

Original article Cañete JD et al. (2008) Clinical significance of synovial lymphoid neogenesis and its reversal after antiTNF- $\alpha$ therapy in rheumatoid arthritis. Ann Rheum Dis [doi:10.1136/ard.2008.089284]

\section{GM-CSF and IL-17 blockade shows beneficial effects in a mouse model of arthritis}

Studies indicate that the cytokines granulocytemacrophage colony stimulating factor (GM-CSF) and interleukin (IL)-17 have important pathogenic roles in rheumatoid arthritis (RA). In vivo experiments have shown that blockade of either cytokine has beneficial effects on disease severity. Plater-Zyberk and colleagues, therefore, studied the effects of GM-CSF and IL-17 double blockade in a mouse model of RA-like disease.

Streptococcal cell wall arthritis was induced in one knee of IL-17 receptor (IL-17R)-knockout C57BI/6 mice and wild-type mice. The mice were then treated with antibodies against GMCSF, IL-1 $\beta$ or tumor necrosis factor (TNF) by intraperitoneal injection.

Mice treated with GM-CSF blockade had significantly decreased joint swelling in their diseased knee; this effect persisted until 4 days after the final injection. TNF blockade with etanercept had no effect on joint swelling, whereas IL-1 $\beta$ blockade caused a modest decrease in swelling. Mice treated with GMCSF or IL-1 $\beta$ blockade had significantly reduced knee cartilage damage. The beneficial effects of GM-CSF blockade on joint swelling and cartilage damage were significantly greater in IL-17R knockout mice than in wild-type mice.

The authors conclude that these results support the hypothesis that blockade of GM-CSF or IL-17 has potential therapeutic benefit in an RA-like arthritis model. Inhibition of both GM-CSF and IL-17 might be especially effective in patients with RA who do not respond to anti-TNF therapy.

Original article Plater-Zyberk C et al. (2008) Combined blockade of GM-CSF and IL-17 pathways potently suppresses chronic destructive arthritis in a TNF $\alpha$-independent mouse model. Ann Rheum Dis [doi:10.1136/ard.2007.085431]

\section{Triptolide and tripdiolide are effective treatments in mice with lupus nephritis}

An extract of Tripterygium wilfordii Hook F (TWHF) is used in traditional Chinese medicine as an antirheumatic herbal remedy. Although the unpurified extract is reportedly an effective treatment for lupus nephritis in mice, the effects of triptolide and tripdiolide, thought to be the two most therapeutically active components of the extract, have not been studied. Tao and colleagues, therefore, investigated the effects of these two diterpenoids when administered separately to mice with established lupus nephritis.

At the age of 29 weeks, NZB $\times N Z W F_{1}$ mice were randomly assigned to treatment with $6 \mu \mathrm{g}$ 